\title{
Fatty acid synthase downregulation contributes to acute lung injury in murine diet-induced obesity
}

\author{
Maria Plataki, ${ }^{1,2}$ LiChao Fan, ${ }^{1}$ Elizabeth Sanchez, ${ }^{1}$ Ziling Huang, ${ }^{1}$ Lisa K. Torres, ${ }^{1}$ Mitsuru Imamura, ${ }^{1}$ \\ Yizhang Zhu, ${ }^{1}$ David E. Cohen, ${ }^{3}$ Suzanne M. Cloonan, ${ }^{1}$ and Augustine M.K. Choi ${ }^{1,2}$ \\ 'Division of Pulmonary and Critical Care Medicine, Joan and Sanford I. Weill Department of Medicine, Weill Cornell Medical \\ College, New York, New York, USA. ${ }^{2}$ NewYork-Presbyterian Hospital/Weill Cornell Medical Center, New York, New York, \\ USA. ${ }^{3}$ Division of Gastroenterology and Hepatology, Joan and Sanford I. Weill Department of Medicine, Weill Cornell \\ Medical College, New York, New York, USA.
}

The prevalence of obesity is rising worldwide, and obese patients constitute a specific population in the intensive care unit. Acute respiratory distress syndrome (ARDS) incidence is increased in obese patients. Exposure of rodents to hyperoxia mimics many of the features of ARDS. In this report, we demonstrate that high-fat diet-induced obesity increases the severity of hyperoxic acute lung injury in mice in part by altering fatty acid synthase (FASN) levels in the lung. Obese mice exposed to hyperoxia had significantly reduced survival and increased lung damage. Transcriptomic analysis of lung homogenates identified Fasn as one of the most significantly altered mitochondria-associated genes in mice receiving a $60 \%$ compared with $10 \%$ fat diet. FASN protein levels in the lung of highfat diet mice were lower by immunoblotting and immunohistochemistry. Depletion of FASN in type II alveolar epithelial cells resulted in altered mitochondrial bioenergetics and more severe lung injury with hyperoxic exposure, even upon administration of a $60 \%$ fat diet. This is the first study to our knowledge to show that a high-fat diet leads to altered FASN expression in the lung, and that both a high-fat diet and reduced FASN expression in alveolar epithelial cells promote lung injury.

Conflict of interest: AMKC is a cofounder, stockholder, and serves on the Scientific Advisory Board for Proterris, which develops therapeutic uses for carbon monoxide. AMKC also has a use patent on CO. AMKC served as a consultant at Teva Pharmaceuticals in July 2018.

Copyright: () 2019, American Society for Clinical Investigation.

Submitted: January 30, 2019

Accepted: July 2, 2019

Published: August 8, 2019

Reference information: /CI Insight. 2019;4(15):e127823.

https://doi.org/10.1172/jci.

insight.127823.

\section{Introduction}

The prevalence of obesity is increasing worldwide (1). In 2015 to 2016 obesity affected $39.8 \%$ of the adults in the United States (2). In particular, obesity is a major risk factor for the development of several respiratory diseases including lung injury (3) and obese patients constitute a specific population in the intensive care unit (ICU) $(4,5)$. Acute respiratory distress syndrome (ARDS) represents a final common pathway of lung inflammation and injury from multiple etiologies and is characterized by the acute onset of hypoxemia with bilateral lung opacities that cannot be explained by cardiac failure or hydrostatic pulmonary edema (6). ARDS constitutes $10.4 \%$ of total ICU admissions (7). Around $20 \%$ of the patients admitted to the ICU are obese (8-10), and obese patients are at greater risk of developing ARDS $(11,12)$. Obese compared with nonobese ARDS patients have distinct respiratory system physiology, which may in turn alter their response to specific treatments $(13,14)$. Furthermore, the relationship between obesity and mortality in these patients appears to be complex $(15,16)$.

Acute hypoxemic respiratory failure in ARDS is secondary to diffuse alveolar damage with epithelial and endothelial injury, alveolar inflammation, altered capillary barrier properties, fibroproliferation, and microvascular coagulopathy, leading to the exudation of protein-rich edema fluid into the alveolar space $(17,18)$. Exposure to high concentrations of oxygen has been shown to cause in various animal species pulmonary toxicity that mimics the clinical and pathologic features of acute lung injury in patients with ARDS $(19,20)$. Even though critically ill patients are often treated with high levels of oxygen for prolonged periods of time, the detrimental effects of hyperoxia remain poorly studied, and what constitutes a "safe" inspired oxygen concentration in this population remains to be determined $(21,22)$.

In the present study, we evaluated the effects of diet-induced obesity on acute lung injury in a murine model of hyperoxic lung injury and investigated the potential mechanisms implicated in the differential response in the setting of a high-fat diet. 


\section{Results}

Twelve weeks of high-fat diet results in weight gain and signs of metabolic dysfunction in a murine model of obesity. We first established a murine model of obesity by utilizing a diet in which $60 \%$ of the calories are derived from fat in the form of lard. After 12 weeks, the weight of C57BL/6J mice fed a $60 \%$ fat diet was significantly higher than that of mice fed a $10 \%$ fat diet $(46.7 \pm 0.85$ vs. $30.9 \pm 0.54 \mathrm{~g}, P<0.0001)$ (Figure $1 \mathrm{~A}$ ). Mice fed a high-fat diet also had significantly higher serum cholesterol $(236.5 \pm 16.1 \mathrm{vs.} 121.5 \pm 11.5 \mathrm{mg} /$ $\mathrm{dL}, P<0.0001)$ and fasting glucose $(213.5 \pm 7.5 \mathrm{vs} .157 .1 \pm 7.1 \mathrm{mg} / \mathrm{dL}, P<0.0001)$ compared with mice with access to a lower-fat diet (Figure $1, \mathrm{~B}$ and $\mathrm{C}$ ).

High-fat diet is associated with reduced survival and increased lung injury after exposure to hyperoxia. To study the effects of obesity on hyperoxic acute lung injury, after 12 weeks of high-fat or low-fat diet, we exposed male $\mathrm{C} 57 \mathrm{BL} / 6 \mathrm{~J}$ mice to greater than $95 \% \mathrm{O}_{2}$ or room air. High-fat diet mice experienced increased mortality under hyperoxic conditions compared with $10 \%$ fat diet mice $(P<0.0001)$ (Figure 2A). High-fat diet and control mice were also exposed to hyperoxia or room air for 48 hours, and bronchoalveolar lavage fluid (BALF) and lung tissue for histology was collected as described in the methods. Increased capillary permeability causes leak of plasma protein into the alveolar space, which in turn results in lung edema that contributes to the hypoxemia observed in acute lung injury. Analysis of BALF showed increased levels of protein $(2.12$ \pm 0.14 vs. $0.97 \pm 0.18 \mathrm{mg} / \mathrm{mL}, P<0.001$, Figure $2 \mathrm{~B}$ ) and $\mathrm{IgM}, 2267 \pm 658$ vs. $473 \pm 221 \mathrm{ng} / \mathrm{mL}, P<0.05$, Figure 2C) in the obese compared with control mice under hyperoxia. Claudin-18, a member of a family of transmembrane proteins that are required for tight junction formation, is the only known lung-specific tight junction protein and is highly expressed in alveolar epithelial cells (23-25). Claudin-18 expression by Western blot was higher in the lungs of obese compared with control mice after hyperoxia $(P<0.01$, Supplemental Figure 1A; supplemental material available online with this article; https://doi.org/10.1172/jci. insight.127823DS1), similar to other studies that have shown barrier deregulation and claudin switching with high-fat diet in other organs (26). Lungs were subjected to H\&E staining. Hyperoxia-induced morphological alterations, including alveolar septal thickening, vascular congestion, and intraalveolar erythrocytes, were more pronounced in the mice receiving the $60 \%$ fat diet when compared with mice receiving the $10 \%$ fat diet (Figure 2D). High-fat diet mice also had increased free fatty acids in the BALF after hyperoxia $(0.80 \pm 0.21 \mathrm{vs}$. $0.002 \pm 0.002 \mathrm{nmol} / 50 \mu \mathrm{L}, P<0.05$, Figure $2 \mathrm{E})$. There was a trend for increased number of cells and BALF macrophages with hyperoxia in the control group $\left(0.48 \pm 0.19 \times 10^{5} \mathrm{vs} .1 .18 \pm 0.15 \times 10^{5}\right.$ total cells $/ \mathrm{mL}$ and $0.46 \pm 0.17 \times 10^{5}$ vs. $1.09 \pm 0.15 \times 10^{5}$ macrophages $/ \mathrm{mL}$, nonsignificant), but there was no significant difference between the obese and control mice (Supplemental Figure 1, B and C). BALF interleukin-6 (IL-6) was clearly increased in obese compared with control mice after hyperoxia, even though it did not reach statistical significance ( $75.7 \pm 37.5$ vs. $2.6 \pm 1.2 \mathrm{pg} / \mathrm{mL}$, Supplemental Figure 1D), and TNFA was undetectable. It is well known that cell death is a prominent feature of hyperoxic lung injury (27). To examine whether cell death was different between diet groups, we measured lactate dehydrogenase (LDH) levels in BALF and performed TUNEL staining of paraffin-fixed lung tissue. Both BALF LDH activity (7.9 \pm 1.6 vs. $15.4 \pm 0.9$ relative levels, $P<0.01)$ and TUNEL-positive cells $(0.07 \pm 0.01$ vs. $0.25 \pm 0.04$ positive cells/number of alveoli, $P<$ 0.01 ) were significantly higher in the high-fat versus the low-fat diet group (Figure $2, \mathrm{~F}-\mathrm{H}$ ). Proliferating cell nuclear antigen (PCNA) expression, indicative of cell proliferation, was not different under hyperoxic conditions between high and low-fat diet lungs (Supplemental Figure 1E). In aggregate, these results indicate that high-fat diet-induced obesity is associated with exaggerated hyperoxic acute lung injury.

To further investigate the relative importance of obesity versus increased fat intake specifically to the increased susceptibility to hyperoxia, we subjected B6.Cg-Lep ${ }^{o b} / \mathrm{J}(o b / o b)$ mice and heterozygous controls $(o b /+)$ to 48 hours of hyperoxia. Lack of leptin in these mice leads to marked early-onset obesity, insulin resistance, and more marked hyperglycemia than the high-fat diet-induced obesity model $(28,29)$. As expected, at 13 weeks of age $o b / o b$ mice had significantly increased weight compared with their heterozygous littermates $(53.2 \pm 1.9 \mathrm{~g}$ vs. $30.9 \pm 0.5 \mathrm{~g}, P<0.0001$, Supplemental Figure $2 \mathrm{~A})$. In contrast to the highfat diet mice, however, $o b / o b$ mice after hyperoxia appeared protected, with BALF protein levels significantly lower than in $o b /+$ mice and similar to those of room air controls $(0.19 \pm 0.01 \mathrm{vs} .0 .48 \pm 0.07 \mathrm{mg} / \mathrm{mL}, P$ $<0.01$, Figure 2I). BALF cell count was also higher in $o b /+$ compared with $o b / o b$ mice after hyperoxia (3.9 $\pm 0.39 \times 10^{5}$ vs. $2.02 \pm 0.28 \times 10^{5}$ total cells $/ \mathrm{mL}, P<0.05$, Supplemental Figure $2 \mathrm{~B}$ ).

Fatty acid synthase is reduced in the lungs of mice receiving high-fat diet and further reduced with hyperoxia. To explore potential mechanisms responsible for the increased sensitivity of obese mice to hyperoxic acute lung injury, we harvested lung tissues for gene expression profiling. Mitochondria play a central role in 

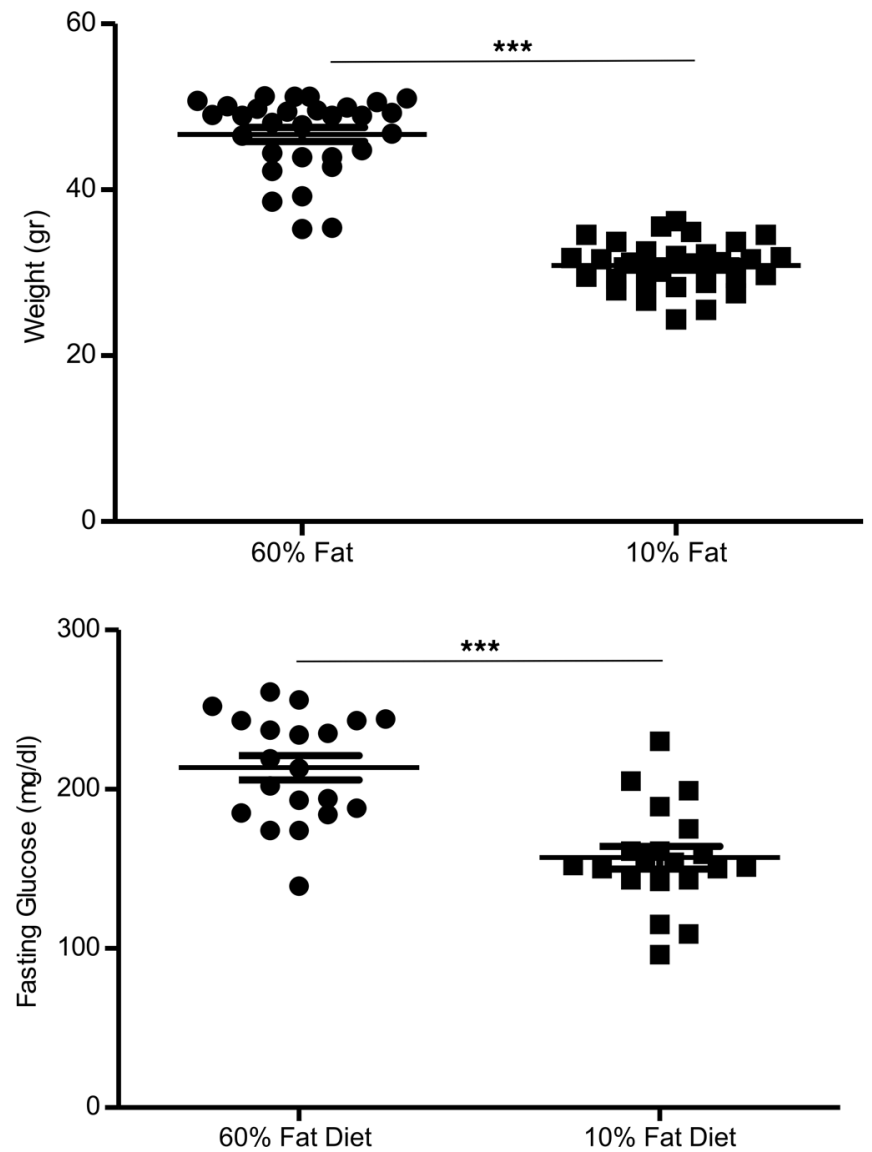

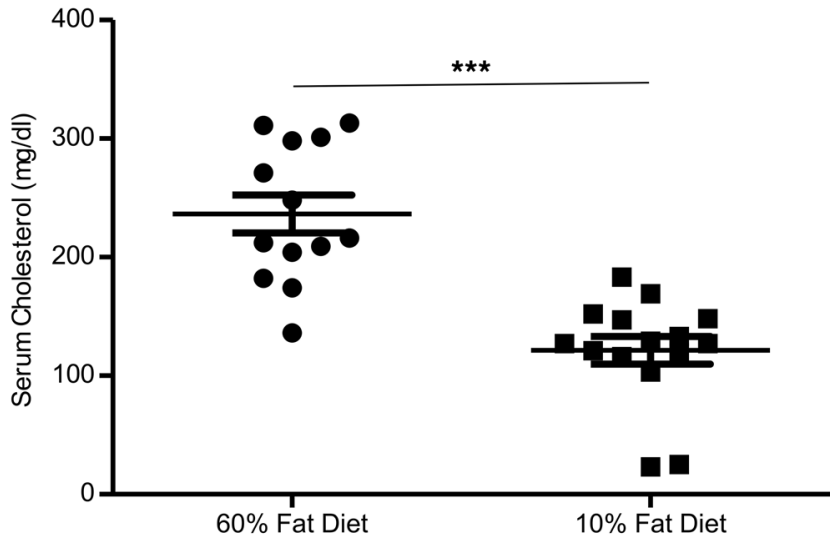

Figure 1. Diet with $60 \%$ of the calories derived from fat-induced weight gain and hypercholesterolemia in a murine model of obesity. (A) Weight ( $n=30$ per group, Mann-Whitney $U$ test, $\left.{ }^{* *} P<0.0001\right)$. (B) Serum cholesterol (mg/dL, $n=13$ in $60 \%$ fat and $n=15$ in 10\% fat group, Mann-Whitney $U$ test, $\left.{ }^{* * *} P<0.0001\right)$. (C) Fasting glucose $(\mathrm{mg} / \mathrm{dL}, n=20$ per group, Mann-Whitney $U$ test, $\left.{ }^{* * *} P<0.0001\right)$ of $[57 \mathrm{BL} / 6$ ) male mice (18 weeks old) receiving $60 \%$ fat or an ingredient-matched $10 \%$ fat diet for 12 weeks. Data are expressed as mean \pm SEM.

energy production, their activity may change in response to nutrients (30), and mitochondrial alterations in the alveolar epithelium are implicated in both hyperoxic lung injury and ARDS $(31,32)$. We therefore focused on the differential lung expression of genes associated with mitochondrial function by comparing the murine MitoCarta gene list, an inventory of genes encoding proteins with mitochondrial localization, with the list of genes that were significantly different between the $60 \%$ and $10 \%$ fat diet mice (Figure $3 \mathrm{~A}$ ). The fatty acid synthesis pathway was represented by fatty acid synthase (Fasn) and acetyl-CoA carboxylase (Acaca) genes, and we identified Fasn as one of the genes significantly reduced in mice receiving a $60 \%$ versus $10 \%$ fat diet and further reduced with hyperoxia (adjusted $P<0.05$, Figure 3B). We focused on FASN because very little is known about the function of FASN in the lung outside the context of lung cancer, and dietary intake has been shown to affect FASN levels in other tissues (33). To determine whether a high-fat diet also affects FASN protein expression in the lung, we performed immunoblotting of lung tissues. Similar to RNA-Seq, FASN protein expression levels were lower in the high-fat diet groups $(P<0.05$, Figure $3 C$ ). There was no significant difference in FASN levels in $o b / o b$ compared with $o b /+$ mice (Supplemental Figure $2 \mathrm{C}$ ). We further examined FASN protein expression by IHC and found a striking reduction in staining in the high-fat compared with the low-fat diet group (Figure 3D).

Mice deficient in FASN in alveolar epithelial cells are more susceptible to lung injury after hyperoxic exposure. Whole-body knockout of FASN causes embryonic lethality in mice (34). To explore whether FASN genetic deletion affects lung response, we employed genetically engineered mice with conditional gene targeting using the Cre/loxP recombinase system. Sftpc $C^{C r E R T 2+/+}$ mice, in which Cre expression is under the control of the surfactant protein promoter and therefore Cre is selectively expressed in alveolar epithelial cells (35), were crossed with FASN ${ }^{\text {loxp/loxp }}$ mice, in which FASN is flanked by loxP sequences (36). To generate mice with FASN deletion specifically in alveolar epithelial cells, 6 daily intraperitoneal tamoxifen injections were given to male FASN ${ }^{\text {loxploxp }} S f t p c^{\text {CreERT2+/- }}$ mice $\left(\right.$ FaSn $^{\text {iAAEC2 }}$ ) to induce recombination by CreERT2. Sftpc CreERT2+/mice (control), also tamoxifen injected, were used as controls. Immunoblotting of lung tissue confirmed that FASN expression was minimal in the lungs of Fasn ${ }^{i \triangle A E C 2}$ mice relative to controls (Figure 4A). Fasn ${ }^{\text {iLAEC2 }}$ mice had increased weight compared with control mice ( $28.0 \pm 0.6$ vs. $25.8 \pm 0.5 \mathrm{~g}, P<0.05$, Figure $4 \mathrm{~B})$. 
A

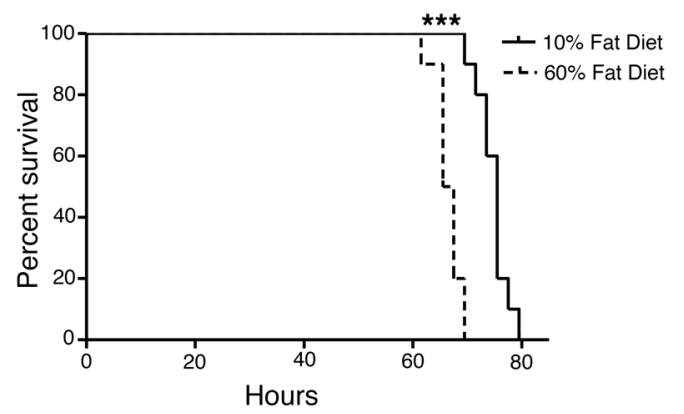

D

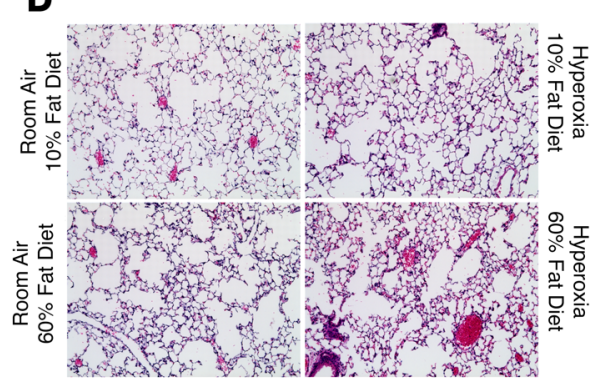

$\mathbf{F}$

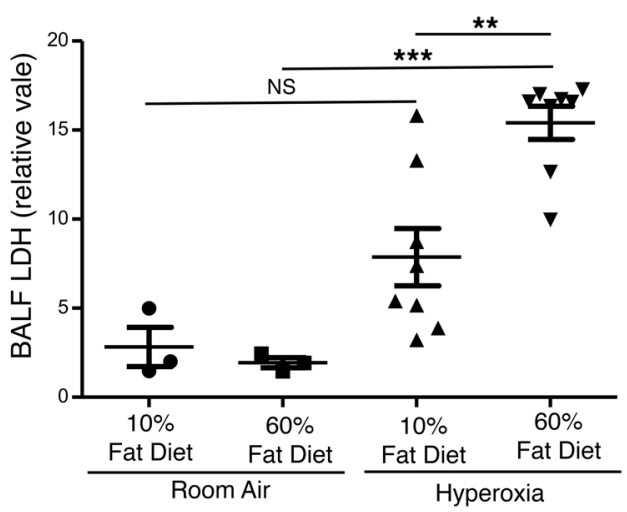

H

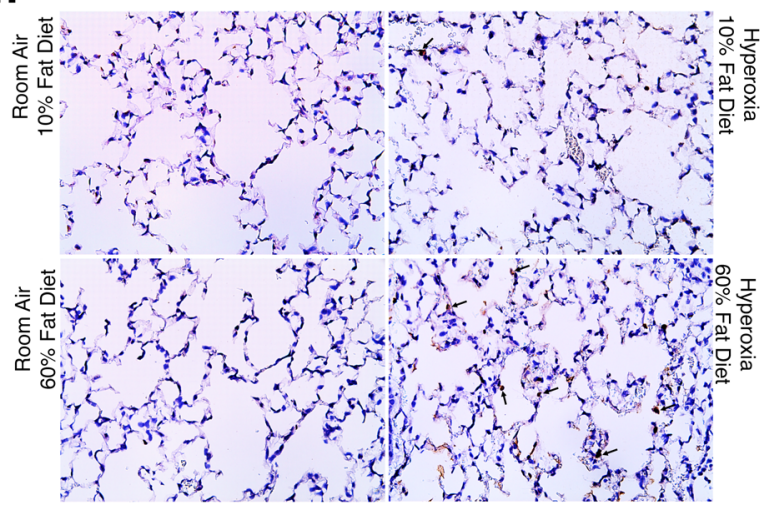

B

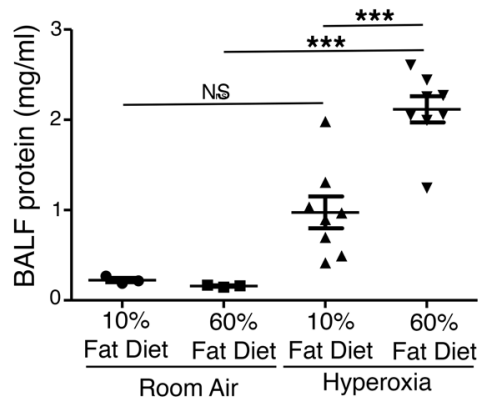

E

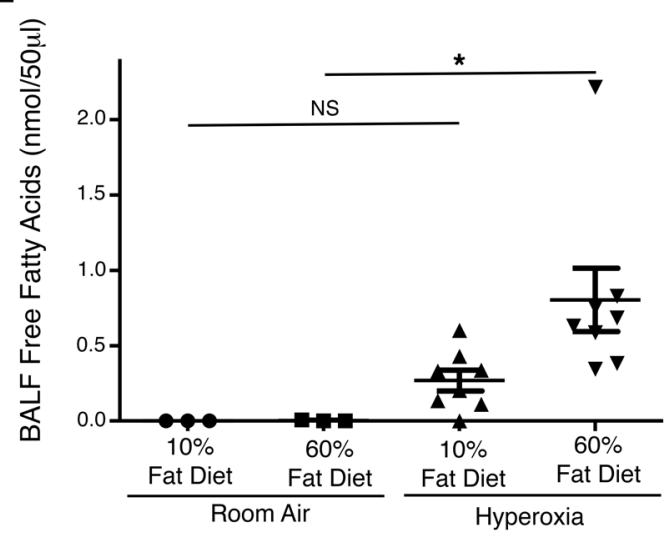

G

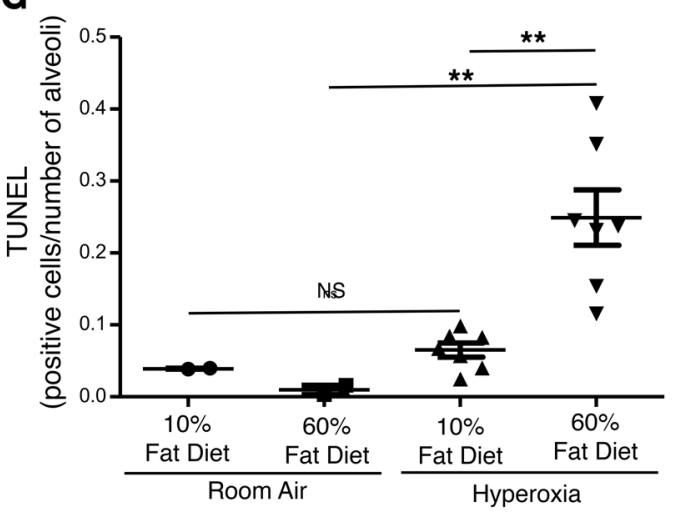

I

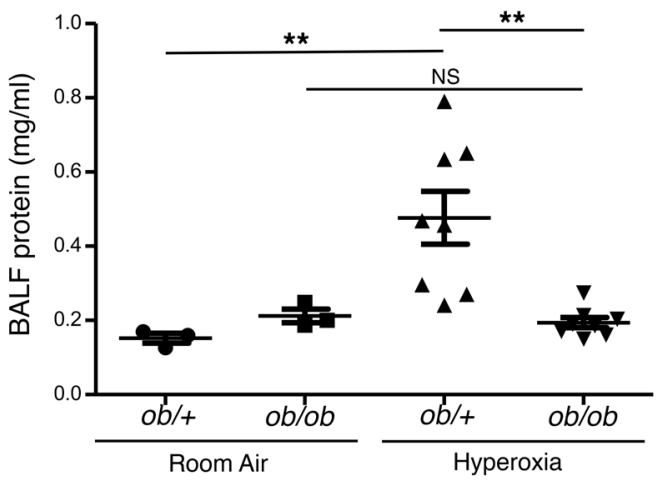


Figure 2. High-fat diet is associated with reduced survival and increased lung injury after exposure to hyperoxia. (A) Representative survival curve of [57BL/6) mice fed $60 \%$ versus ingredient-matched $10 \%$ fat diet exposed to $>95 \%$ oxygen ( $n=10$ per group, log-rank test, $\left.{ }^{* * *} P<0.0001\right)$. (B) Bronchoalveolar lavage fluid (BALF) protein levels from mice fed $60 \%$ and $10 \%$ fat diet after 48 hours of exposure to $>95 \%$ oxygen or room air ( $\mathrm{mg} / \mathrm{mL} n=3$ per group for room air and $n=8$ per group for hyperoxia, ANOVA with Tukey's post hoc correction, ${ }^{* *} P<0.001$, similar results were obtained from at least 2 independent experiments). (C) BALF IgM levels from mice fed $60 \%$ and $10 \%$ fat diet after 48 hours of exposure to $>95 \%$ oxygen or room air ( $\mathrm{ng} / \mathrm{mL}, n=3$ per group for room air and $n=8$ per group for hyperoxia, ANOVA with Tukey's post hoc correction, ${ }^{*} P<0.05$, similar results were obtained from at least 2 independent experiments). (D) Representative image of H\&E-stained lungs ( $n=2$ per group for room air and $n=7$ per group for hyperoxia; original magnification, $\times 20$ ). (E) BALF free fatty acid levels after 48 hours of hyperoxia or room air ( $n$ mol/50 $\mu \mathrm{L}, n=3$ per group for room air and $n=8$ per group for hyperoxia, ANOVA with Tukey's post hoc correction, $\left.{ }^{*} P<0.05\right)$. (F) BALF lactate dehydrogenase (LDH) levels after 48 hours of hyperoxia or room air (relative value, $n=3$ per group for room air and $n$ $=8$ per group for hyperoxia, ANOVA with Tukey's post hoc correction, ${ }^{* *} P<0.001,{ }^{* *} P<0.01$ ). (C) TUNEL expressed as positive cells per number of alveoli after 48 hours of hyperoxia or room air ( $n=2$ per group for room air and $n=7$ per group for hyperoxia, ANOVA with Tukey's post hoc correction, ${ }^{* *} P<0.01$ ). (H) Representative image of TUNEL-stained lungs (original magnification, $x 40$; arrows highlight positive cells). (I) BALF protein levels from B6.Cg-Lep ${ }^{o b} / J$ (ob/ ob) mice and heterozygous controls (ob/+) after 48 hours of exposure to $>95 \%$ oxygen or room air $(\mathrm{mg} / \mathrm{mL}, n=3$ per group for room air and $n=8$ per group for hyperoxia, ANOVA with Tukey's post hoc correction, ${ }^{* *} P<0.01$ ). Data are expressed as mean \pm SEM.

To examine whether genetic deletion of FASN in alveolar epithelial cells affects lung injury at baseline or after hyperoxic exposure, Fasn ${ }^{i A E C 2}$ and control mice were exposed to room air or hyperoxia for 48 hours, and BALF was collected as described in Methods. There was no significant difference in BALF protein or IgM between Fasn $n^{i A E C 2}$ and controls under room air, but after hyperoxia FaSn ${ }^{i A E C 2}$ mice had significantly increased alveolar permeability by both indices compared with control mice (protein $0.54 \pm 0.04$ vs. $0.41 \pm$ $0.03 \mathrm{mg} / \mathrm{mL}, P<0.05$, Figure 4C; and IgM $109 \pm 7$ vs. $83 \pm 6 \mathrm{ng} / \mathrm{mL}, P<0.05$, Figure 4D). Our results also revealed that, after hyperoxia, mice deficient in FASN in alveolar epithelial cells had significantly higher BALF LDH levels ( $7.2 \pm 0.8$ vs. $4.9 \pm 0.4, P=0.05$, Figure $4 \mathrm{E})$ and increased lung injury by histological analysis (Figure $4 \mathrm{~F}$ ). There was no significant difference in BALF total cell count and macrophage numbers between the groups (Supplemental Figure 3, A and B). BALF IL-6 was increased with hyperoxic exposure in both groups, and there was no significant difference between Fasn ${ }^{i A E C 2}$ and control mice (15.8 \pm 1.6 vs. $18.3 \pm 3.4 \mathrm{pg} / \mathrm{mL}$, nonsignificant, Supplemental Figure 3C). TNFA was undetectable. We also performed a

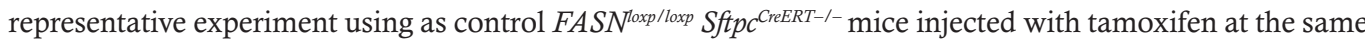
time as the Fasn ${ }^{i A E C 2}$ mice. With hyperoxic exposure there was a more significant increase in BALF protein

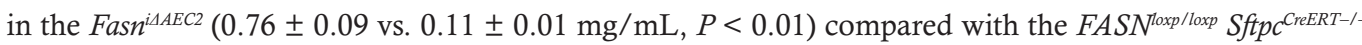
mice $(0.69 \pm 0.12$ vs. $0.16 \pm 0.03 \mathrm{mg} / \mathrm{mL}, P<0.05)$ (Supplemental Figure 3D). These data indicate that mice with selective FASN deletion in alveolar epithelial cells have increased injury and alveolar leakage, and an exacerbated response to hyperoxia compared with control mice.

To determine the effect of FASN deletion on mitochondria, we examined the levels of oxidative phosphorylation complexes and representative proteins from the mitochondrial outer membrane, intermembrane space, inner membrane, and matrix space in isolated lung alveolar epithelial type II (AEC2) cells from Fasn ${ }^{i A E C 2}$ and control mice by Western blot analysis (Figure 4G and Supplemental Figure 3E). All proteins were reduced in AEC2 cells from Fasn ${ }^{i A E C 2}$ mice when compared with controls, indicating that FASN downregulation may be associated with mitochondrial alterations. Using the Agilent Seahorse XFe96 analyzer, mitochondrial functional analysis was performed using inhibitors of the electron transport chain to measure oxygen consumption rate (OCR), a marker of oxidative phosphorylation, and extracellular acidification (ECAR), a surrogate of glycolysis. We determined the OCR of basal and maximal respiration, as well as the spare respiratory capacity, in isolated AEC2 cells from Fasn ${ }^{i A E C 2}$ and control mice exposed to room air or hyperoxia for 48 hours. As anticipated, hyperoxic exposure resulted in impaired mitochondrial metabolism in control AEC2 cells, characterized by a loss in spare respiratory capacity and maximal respiration (Supplemental Figure 3F). Control AEC2 cells also displayed increased extracellular acidification in response to hyperoxia, indicative of a higher level of glycolysis. Most importantly, under hyperoxic conditions, AEC2 cells from Fasn ${ }^{i A E C 2}$ mice had more severe alterations in mitochondrial respiration, with reduced basal and maximal respiration, minimal spare respiratory capacity, and impaired switch to glycolysis compared with AEC2 cells from control mice (Figure $4 \mathrm{H}$ and Supplemental Figure 3F). Phenotypic differences between hyperoxic Fasn ${ }^{i A E C 2}$ and control cells were evident, with Fasn ${ }^{i A E C 2}$ cells remaining in a more quiescent state (Supplemental Figure 3F). These results suggest that FASN deficiency alters mitochondrial bioenergetics in AEC2 cells, leading to mitochondrial dysfunction and impaired metabolic potential under stress conditions.

Mice deficient in FASN in alveolar epithelial cells are more prone to lung injury even when they receive a high-fat diet. Our results so far suggest that obesity from a high-fat diet increases the severity of murine acute lung 

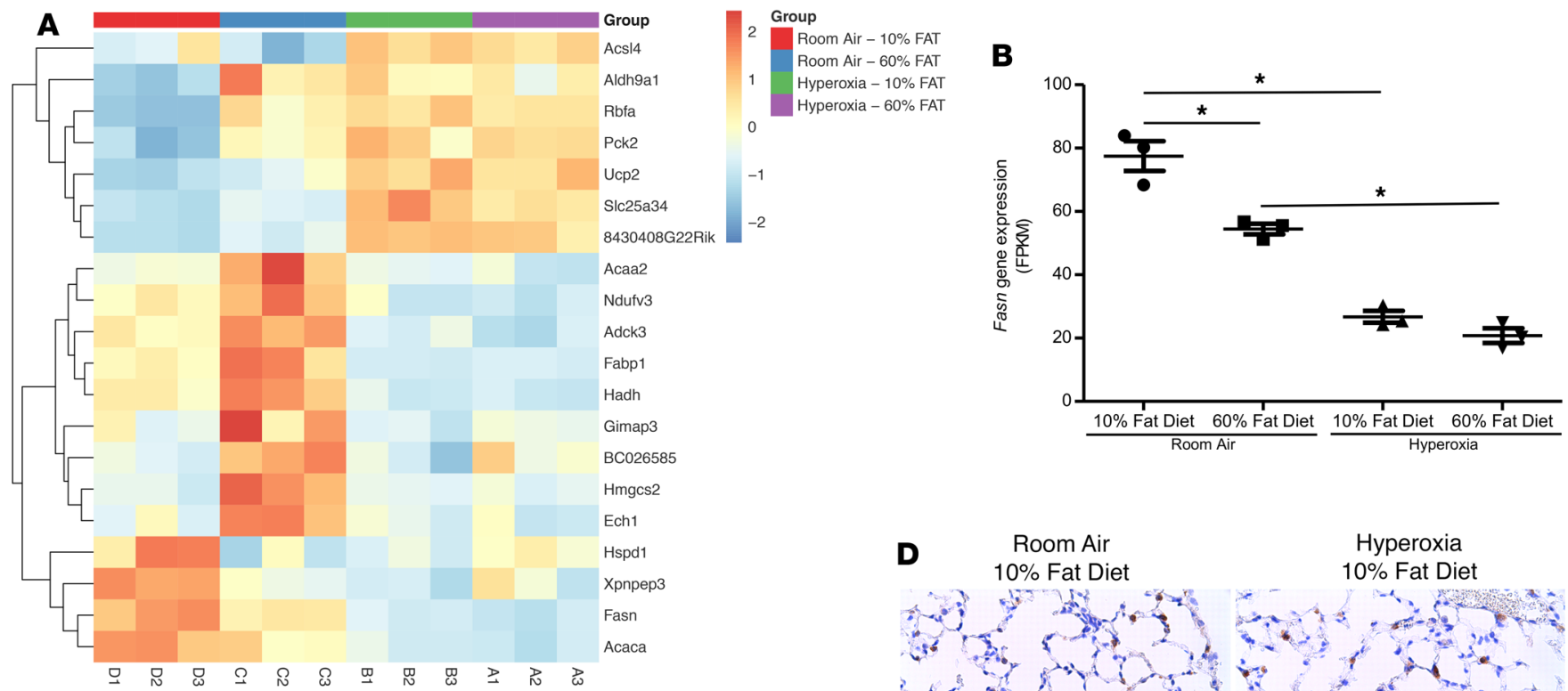

C Room Air Hyperoxia

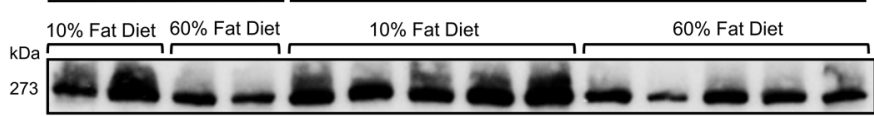

FASN

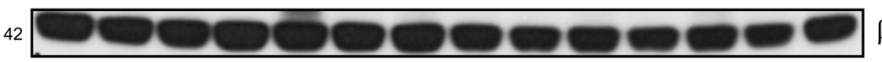
$\beta-\operatorname{actin}$

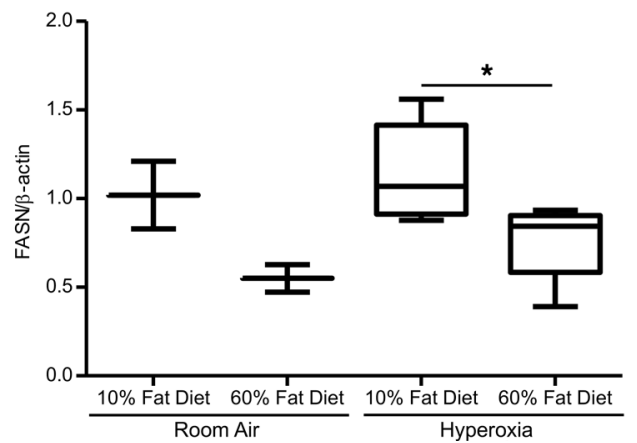

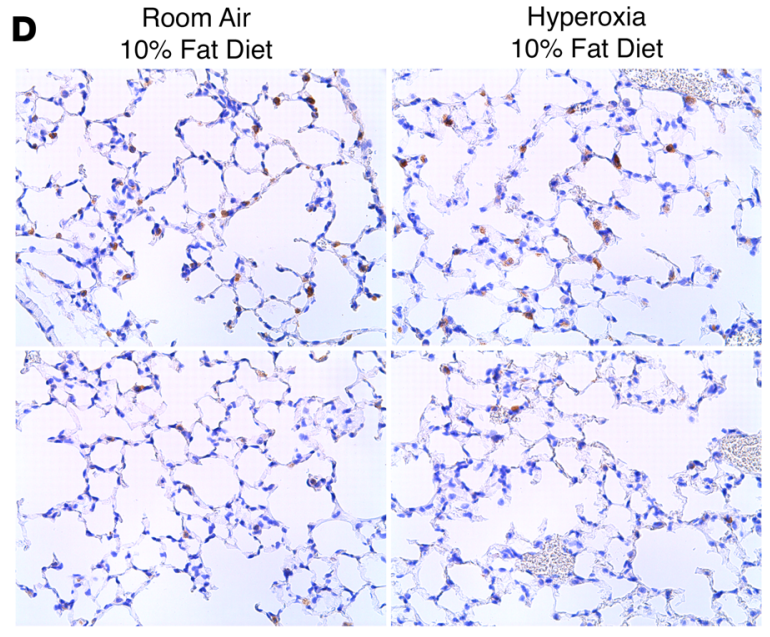

Room Air $60 \%$ Fat Diet
Hyperoxia $60 \%$ Fat Diet

Figure 3. Fatty acid synthase is reduced in the lungs of mice receiving high-fat diet and further reduced with hyperoxia. (A) Differential lung expression by RNA-Seq of genes associated with mitochondrial function as determined by per mouse MitoCarta (an inventory of genes encoding proteins with mitochondrial localization) that were significantly different between the $60 \%$ and $10 \%$ fat diet mice. (B) Relative gene expression levels of fatty acid synthase (Fasn) from RNA-Seq of whole-lung homogenates from mice fed $60 \%$ and $10 \%$ fat diet exposed for 48 hours to hyperoxia or room air ( $n$ = 3 mice per group; the fold change of fragments per kilobase of transcript per million (FPKM) fragments sequenced was calculated relative to control, Benjamini-Hochberg method, *adjusted $P<0.05$ ). (C) Western blot analysis for FASN in lungs with $\beta$-actin loading control. Densitometry analysis shown on the bottom ( $n=2$ per group for room air and $n=5$ per group for hyperoxia, Mann-Whitney $U$ test, ${ }^{*} P<0.05$ ). (D) Representative section of FASN-stained lungs ( $n=2$ per group for room air and $n=7$ per group for hyperoxia; original magnification, $x 40$ ). Data are expressed as mean \pm SEM.

injury in the setting of hyperoxia, and a high-fat diet induces downregulation of FASN in lung tissue. Also, mice with FASN genetic deletion in alveolar epithelial cells that receive regular diet are more susceptible to acute lung injury. FASN reduction, however, after a high-fat diet may represent a compensatory reaction rather than a damage-inducing mechanism, as is the case in the setting of lack of compensation with exogenous dietary fatty acids. We first established that a $60 \%$ fat diet significantly increases lung tissue free fatty acids $(9.7 \pm 0.5$ vs. $6.2 \pm 0.7 \mu \mathrm{mol} / \mathrm{g}$ lung, $P<0.001$, Figure $5 \mathrm{~A})$. In order to determine whether high-fat diet would rescue the detrimental effects of FASN genetic deletion in alveolar cells, we administered $60 \%$ and $10 \%$ fat diets for 15 weeks in Fasn $n^{i \triangle A E C 2}$ and control mice and exposed them to room air or hyperoxia. Both Fasn ${ }^{i \triangle A E C 2}$ and control mice on the high-fat diet had significantly increased weight compared with mice on the $10 \%$ fat diet, and there was no significant difference in weight between Fasn $n^{i \triangle A E C 2}$ and control mice 
A FasniAaEC2

Control

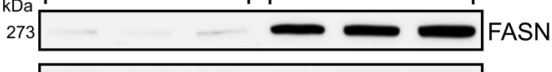

${ }_{42} \longrightarrow{ }_{\beta}-$ actin
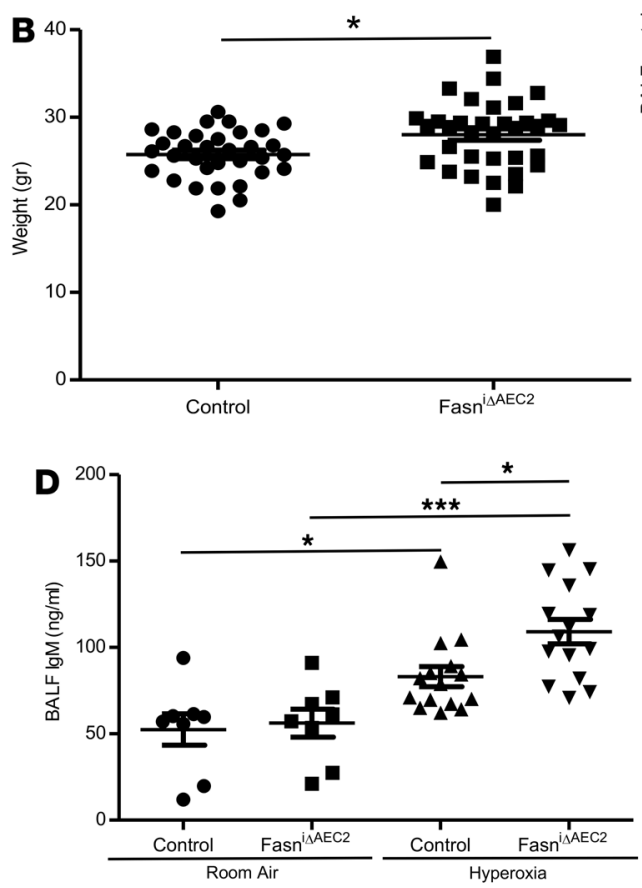

$\mathbf{F}$

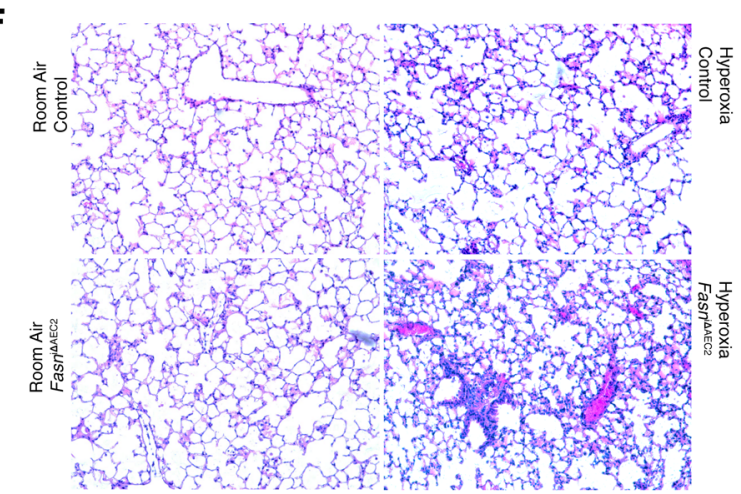

H

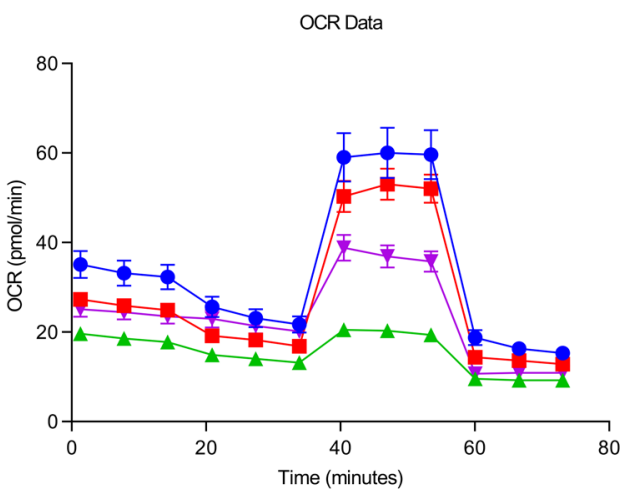

E
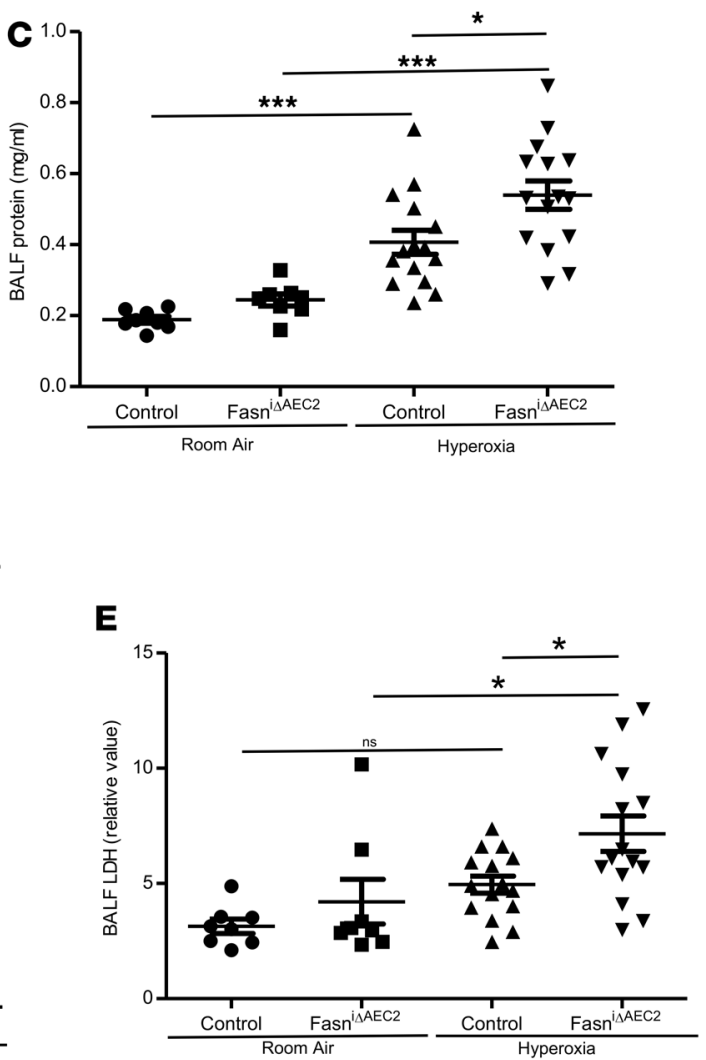

G

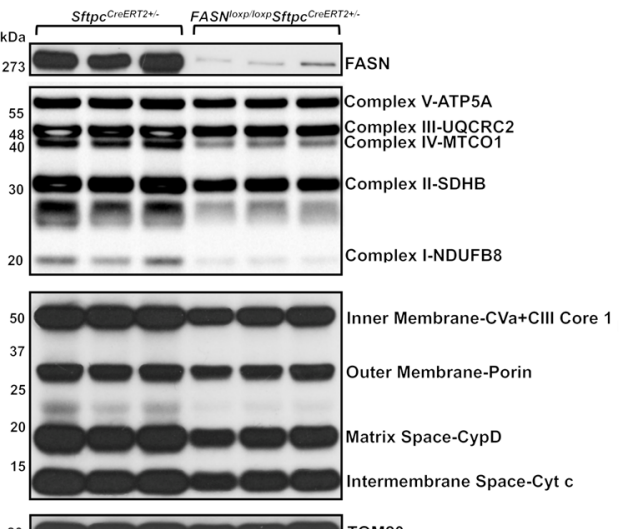

ECAR Data

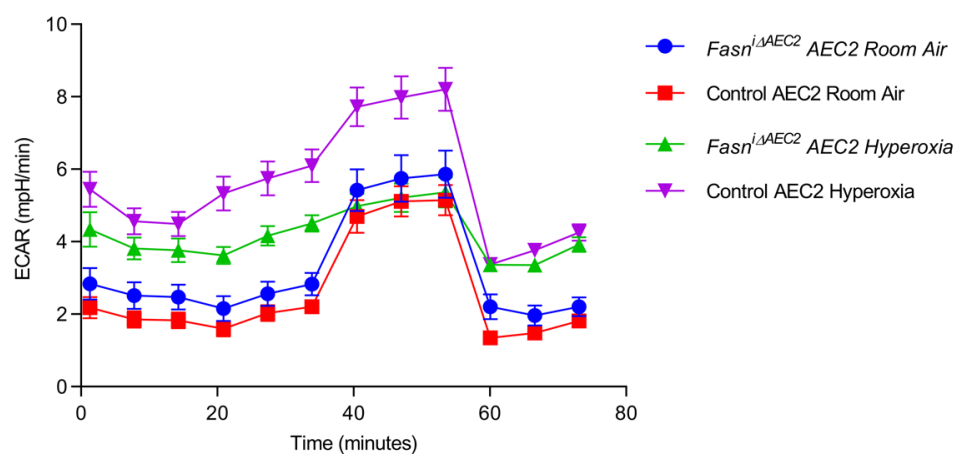


Figure 4. Mice deficient in FASN in alveolar epithelial cells are more susceptible to lung injury after hyperoxic exposure. (A) Western blot analysis

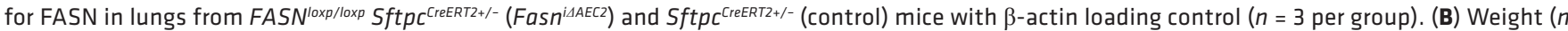
$=33$ for control and $n=34$ for Fasn ${ }^{\text {iAAEC2 }}$ mice, Mann-Whitney $U$ test, $\left.{ }^{*} P<0.05\right)$. (C) Bronchoalveolar lavage fluid (BALF) protein levels from Fasn ${ }^{\text {iAAEC2 }}$ and control mice after 48 hours of exposure to $>95 \%$ oxygen or room air $(\mathrm{mg} / \mathrm{mL}, n=8$ per group for room air and $n=15$ per group for hyperoxia, ANOVA with Tukey's post hoc correction, ${ }^{* *} P<0.001,{ }^{*} P<0.05$; similar results were obtained from at least 2 independent experiments). (D) BALF IgM levels from Fasn ${ }^{\text {iAAEC2 }}$ and control mice after 48 hours of exposure to $>95 \%$ oxygen or room air $(\mathrm{ng} / \mathrm{mL}, n=8$ per group for room air and $n=15$ per group for hyperoxia, ANOVA with Tukey's post hoc correction, ${ }^{* * *} P<0.001$, ${ }^{*} P<0.05$, similar results were obtained from at least 2 independent experiments). (E) BALF lactate dehydrogenase (LDH) levels after 48 hours of hyperoxia or room air (relative value, $n=8$ per group for room air and $n=15$ per group for hyperoxia, ANOVA with Tukey's post hoc correction, ${ }^{*} P<0.05$, similar results were obtained from at least 2 independent experiments). (F) Representative image of H\&E-stained lungs ( $n=3$ per group for room air and $n=5$ per group for hyperoxia; original magnification, $\times 20)$. (C) Alveolar epithelial type II (AEC2) cells were isolated from FasniAAEC2 and control mice, and protein expression was assessed using the Total OXPHOS (ubiquinone oxidoreductase subunit B8 [NDUFB8], succinate dehydrogenase complex iron sulfur subunit B [SDHB], ubiquinol-cytochrome c reductase core protein 2 [UQCRC2], mitochondrially encoded cytochrome c oxidase 1 [MTC01], ATP synthase subunit alpha [ATP5A]) (top) and the mitochondrial Membrane Integrity antibody cocktail (outer membrane-porin, intermembrane space-cytochrome $c$, inner membrane-complex VA and complex III core 1, matrix space-cyclophilin D) (bottom). TOM20 expression was used to confirm equivalent protein input ( $n=3$ per group). (H) Oxygen consumption rate (OCR, left) and extracellular acidification rate (ECAR, right) of isolated AEC2 cells from FASN ${ }^{\text {loxp/loxp }}$ Sftpc $^{\text {CreERT2+/- }}$ (Fasn ${ }^{\text {iAAEC2 }}$ AEC2) and $S f t p c^{\text {CreERT2+/- }}$ mice (Control AEC2) exposed to room air or hyperoxia for 48 hours. All data are raw values (similar results were obtained from 2 independent experiments). Data are expressed as mean \pm SEM.

on the same diet (Figure 5B). When exposed to greater than $95 \%$ oxygen for 48 hours, control mice on a $60 \%$ fat diet had increased lung damage compared with control mice on a 10\% fat diet (BALF protein 1.25 \pm 0.23 vs. $0.61 \pm 0.07 \mathrm{mg} / \mathrm{mL}, P<0.05$ ), but no significant difference compared with Fasn ${ }^{i A E C 2}$ mice on a $10 \%$ fat diet (BALF protein $1.25 \pm 0.23$ vs. $0.87 \pm 0.08 \mathrm{mg} / \mathrm{mL}$, nonsignificant); and Fasn ${ }^{i \triangle A E C 2}$ mice on a $60 \%$ fat diet did not have reduced injury compared with the same mice on a $10 \%$ fat diet (BALF protein 1.1 \pm 0.07 vs. $0.87 \pm 0.08 \mathrm{mg} / \mathrm{mL}$, nonsignificant, Figure $5, \mathrm{C}$ and $\mathrm{D}$ ). These findings indicate that exogenous fatty acid supplementation did not decrease the detrimental effects of FASN deficiency in alveolar epithelial cells in rodents after hyperoxia, suggesting that FASN downregulation may be more than a negative feedback mechanism to regulate fatty acid supply in the setting of a high-fat diet.

\section{Discussion}

This study shows that high-fat diet-induced obesity augments the severity of hyperoxia-induced acute lung injury in mice and leads to decreased FASN expression in the lung. We also found that reduced FASN in alveolar epithelial cells is associated with impaired mitochondrial metabolic potential under stress and increased acute lung injury in mice even in the absence of obesity and external supplementation of fatty acids with high-fat diet did not rescue the phenotype of the FASN deficient mice.

Given the rising prevalence of obesity (37) and the fact that obese patients constitute $15 \%-20 \%$ of the ICU population $(8,9)$, the paucity of data on the impact of obesity on acute lung injury compared with other organs is striking. A few studies suggest that rising BMI is associated with increased risk of ARDS $(11,12)$, as well as increased morbidity and resource utilization among critically ill patients. On the other hand, several meta-analyses and observational studies have failed to show that obese patients have reduced survival compared with normal-weight ICU patients $(15,38,39)$. It has been postulated that this "obesity paradox" could be attributed to preconditioning, with obesity causing a low-grade inflammation that primes the lung for a second insult and/or the ability to better store nutrients to overcome stress conditions $(15,16)$. The interpretation of these observations, however, is confounded by many clinical management-related and obesity-specific physiological factors that are challenging to quantify. Clinicians tend to be concerned that obese patients have the potential for worse outcomes and may admit them earlier in the ICU for closer observation and be stricter with prophylactic interventions such as early mobilization, sedation holds, and ventilatory settings. Obesity poses diagnostic challenges with correctly interpreting or even acquiring chest imaging. Moreover, decreased chest wall compliance predisposes obese patients to hypoxemia from atelectasis, which is often misinterpreted as acute lung injury, but more readily reversible, with fewer complications. Until these issues are systematically addressed no safe conclusion can be drawn about the relationship of obesity with mortality in critical illness and the term "obesity paradox" should be abandoned, as it is misleading (40).

This is the first study to our knowledge to examine the effects of diet-induced obesity on acute lung injury induced by hyperoxia. Several animal studies have previously attempted to decipher the relationship between obesity and acute lung injury. The results of these studies often appear contradictory, but they are not directly comparable, as they use different mouse models of obesity, variable models of acute lung injury, and focus 

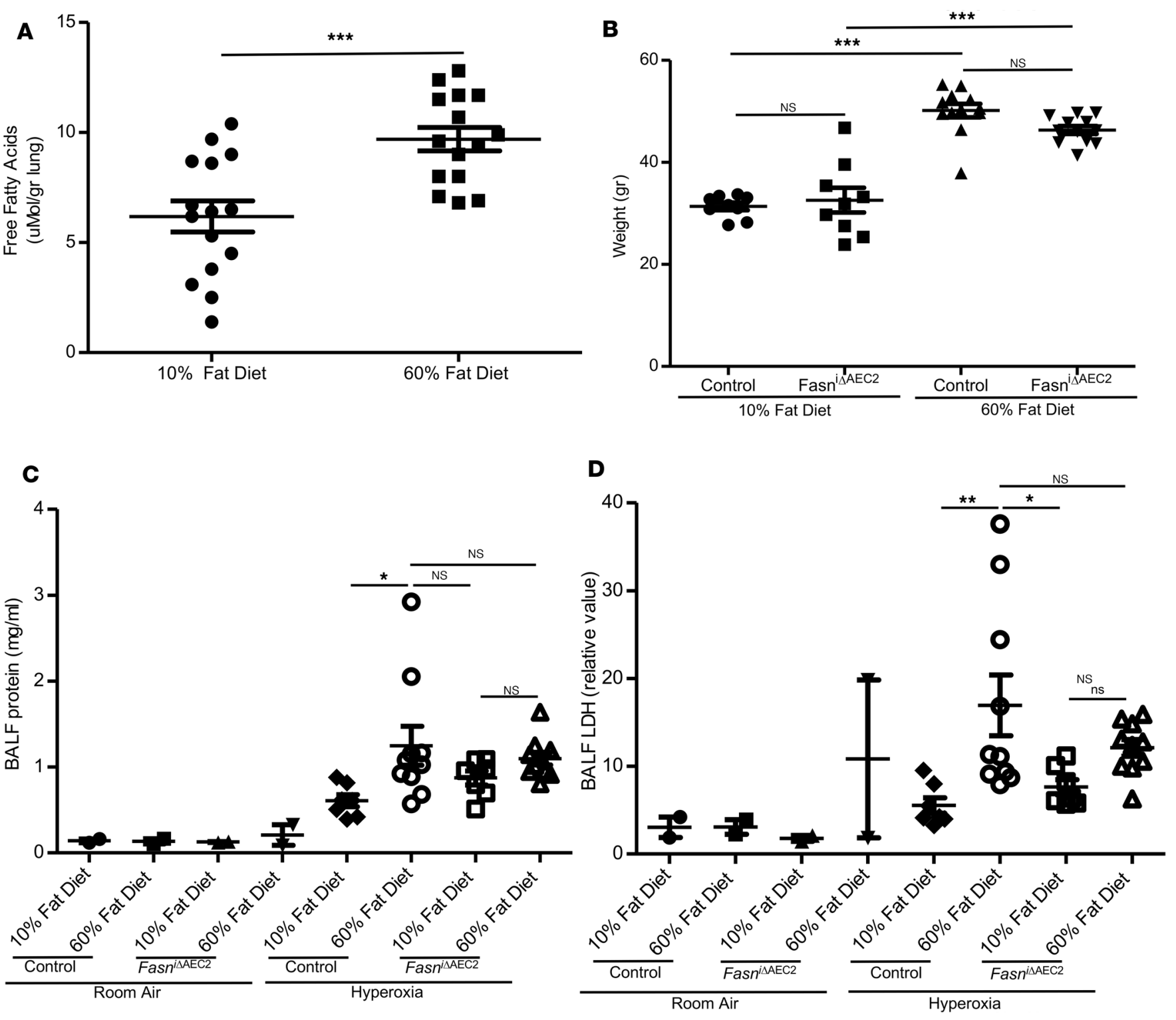

Figure 5. Mice deficient in FASN in alveolar epithelial cells are more prone to lung injury even when they receive high-fat diet. (A) Concentrations of free fatty acids in lipid extracts from lung tissue ( $\mu \mathrm{mol} / \mathrm{g}$ lung, $n=15$ per group, Mann-Whitney $U$ test, ${ }^{* * *} P<0.001$ ). (B) Weight of $F A S N^{10 \times p / l o x p} S f t p c^{\text {CreeERT2+/- }}$

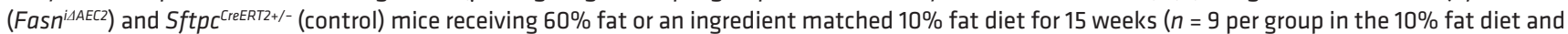
$n=12$ per group in the 60\% fat diet, ANOVA with Tukey's post hoc correction, ${ }^{* *} P<0.001$ ). (C) Bronchoalveolar lavage fluid (BALF) protein levels from Fasn ${ }^{i A E C L}$ and control mice receiving $60 \%$ or $10 \%$ fat diet after 48 hours of exposure to $>95 \%$ oxygen or room air $(\mathrm{mg} / \mathrm{mL}, n=2$ per group for room air, $n=7$ per group for hyperoxia $10 \%$ fat diet, and $n=10$ per group for hyperoxia $60 \%$ fat diet, ANOVA with Tukey's post hoc correction, $\left.{ }^{*} P<0.05\right)$. (D) BALF lactate dehydrogenase (LDH) levels after 48 hours of hyperoxia or room air in Fasn $n^{\text {iAAEC2 }}$ and Control mice receiving $60 \%$ or $10 \%$ fat diet (relative value, $n=2$ per group for room air, $n=7$ per group for hyperoxia 10\% fat diet, and $n=10$ per group for hyperoxia $60 \%$ fat diet, ANOVA with Tukey's post hoc correction, $\left.{ }^{* *} P<0.01,{ }^{*} P<0.05\right)$. Data are expressed as mean \pm SEM.

on different cell types (28). Wilson et al. reported that consumption of a high-fat diet protected mice in terms of alveolar permeability from ventilator-induced lung injury (VILI) using large tidal volumes, independent of leukocyte recruitment and intra-alveolar inflammatory cytokines (41). Guivarch et al. found that fat feeding and obesity were not associated with differences in VILI using lower tidal volumes, but alveolar neutrophil infiltration by histology was increased in obese mice (42). In a different model of acute lung injury, both high-fat diet-fed and leptin-resistant obese mice exhibited reduced lung injury after LPS inhalation (43). Yet obesity from a modified diet exacerbated lung injury after endotracheal LPS instillation in another report (44). We consider hyperoxia a more "physiologically appropriate" model to study acute lung injury in the setting of obesity, as it provides a more uniform exposure to the insult, and it can eliminate some of the shortcomings 
of the other models in the presence of increased weight. Lack of a way to calculate ideal body weight in mice makes standardization of tidal volume challenging. Altered chest wall mechanics can lead to increased atelectasis in obese mice, which may affect both LPS distribution and degree of VILI. Both interventions also necessitate sedation, and increased sedation requirements in obese mice may affect blood pressure and the ensuing lung injury. Bellmeyer et al. reported that leptin-resistant mice exposed to hyperoxia had decreased lung injury compared with WT mice (45). Their investigation was designed primarily to explore the protective effect of diabetes on lung injury, but leptin-resistant mice also exhibited increased weight compared with controls. The leptin-resistant animals, however, had significantly less weight gain (30-35 g compared with $46.7 \pm 0.85$ g) and were younger than the obese mice in our study. Similarly, we found ob/ob mice (deficient in leptin) to be protected from hyperoxic acute lung injury compared with heterozygous littermates in terms of increased alveolar permeability, even though they reached $50 \mathrm{~g}$. This discrepant finding compared with a high-fat diet could be due to leptin-specific mechanisms. High-fat diet-induced obesity in mice is considered an obesity model that is closer to human conditions, because it encompasses many different aspects of the metabolic syndrome. The fact that leptin-resistant and $o b / o b$ mice are resistant to injury may mirror findings in humans, where diabetes is associated with decreased risk of developing ARDS, whereas obesity is associated with increased risk of ARDS, pointing out the importance of the contribution of individual aspects of metabolic syndrome to the final phenotype. Obesity in the clinical setting may be more complex, where individuals with more of a diabetic profile are "protected" from acute lung injury, whereas individuals with higher lipid intake are more susceptible; and the balance between the two dictates the final outcome. A refined carbohydrate diet was found to promote inflammation and redox imbalance in mice after hyperoxia, but alveolar permeability was not measured in that study (46). A major limitation of our model is that we cannot discern whether the injurious effect on acute lung injury is due to the increased weight or the augmented fat intake irrespective of weight, although the findings in the $o b / o b$ mice point to the latter. Another limitation is that although obesity from a high-fat diet may be more clinically relevant than mutant strains, we cannot distinguish between the detrimental effects of the individual elements of metabolic syndrome.

Hyperoxia was associated with increased BALF free fatty acids that were further increased in $60 \%$ fat diet mice. This could represent another index of increased alveolar permeability. Our group, however, has also previously demonstrated increased BALF free fatty acids in mice after ventilator-induced lung injury increased injury with ventilation with fatty acid oxidation inhibition in mice and evidence of impaired fatty acid oxidation in critically ill patients with ARDS. Receptor-interacting protein kinase 3 (RIPK3) may play a role in this association (47). Hyperoxic exposure is also associated with increased RIPK3 expression in the lung $(48,49)$. The findings of impaired fatty acid oxidation in ventilator-induced lung injury may be generalizable to other forms of acute lung injury, especially in the setting of a high supply of exogenous fatty acids that overwhelms mitochondrial uptake ability. The possible contribution of impaired fatty acid oxidation, especially in a high-fat diet setting, in acute lung injury requires more in-depth exploration.

FASN is the enzyme catalyzing the first step of de novo lipogenesis and synthesizes long-chain fatty acids by using acetyl coenzyme A (CoA), malonyl-CoA, and NADPH as reducing equivalent (50). The predominant product is the 16-carbon saturated fatty acid palmitate, but FASN may also produce smaller amounts of shorter-chain fatty acids. These fatty acids can be used for energy storage, membrane assembly and repair, protein palmitoylation, and secretion in the form of lipoproteins. The protein exists as a homodimer of $273 \mathrm{kDa}$ subunits, with each monomer consisting of all 7 required domains for fatty acid synthesis (51). Its tissue distribution is broad with the highest levels in the liver, adipose tissue, and the lung $(50,52)$. Whole-body knockout of FASN is embryonically lethal (34). It has previously been shown that FASN is mainly expressed in AEC2 cells in the lung (53). In this article, we additionally demonstrate that immunoblots from whole lung in which FASN has been specifically deleted from AEC2 cells show almost complete FASN depletion, confirming that FASN is primarily expressed in AEC2 cells. De novo lipogenesis has been shown to be affected by dietary intake in liver and adipose tissue and is altered in diseases such as obesity and cancer $(27,50)$. Our study is the first to our knowledge to examine the effect of a high-fat diet and obesity on FASN expression in the lung. Dietary fat decreases FASN expression in the liver, which decreases de novo lipogenesis when exogenous fats are abundant $(50,54)$. Similarly, we have found that high-fat feeding is associated with reduced FASN expression in lung tissue. Tissue-specific knockout models have implicated FASN in the transmission of signals relevant to metabolic disorders (36). The role of FASN in the lung is largely unexplored. We believe this to be the first study to employ alveolar epithelial cell-specific deletion of FASN to elucidate the role of FASN in acute lung injury. Reduced FASN expression in alveolar 
epithelial cells was associated with increased lung injury and alveolar permeability. This is in accordance with the findings in other tissues. In the intestinal epithelium and endothelium, FASN provides the substrate for palmitoylation of key proteins to maintain tissue integrity $(55,56)$, and the epithelial cells in the colon of tamoxifen-inducible FASN-knockout mice were disordered at the luminal border, suggesting compromise of the intestinal barrier (36). Impaired lipogenesis has also been implicated in increased cell death (36), and Fasn ${ }^{\mathrm{i} \triangle \mathrm{AEC} 2}$ mice did have marginally significantly increased BALF LDH levels compared with controls. We also demonstrated that oxidative phosphorylation complex proteins are reduced in AEC2 cells from Fasn ${ }^{i \triangle A E C 2}$ compared with control mice, and, most importantly, this was associated with impaired metabolic potential under stress, with reduced maximal respiration and spare capacity, as well as impaired switch to glycolysis under hyperoxic conditions. The precise molecular signaling pathways involved in the exaggerated injurious response to hyperoxia in mice deficient in FASN in alveolar epithelial cells will be investigated in future studies. Insulin is also known to affect FASN in other tissues, and a limitation of the present investigation is that we did not explore insulin levels.

In conclusion, our findings highlight the critical role of increased fat intake and obesity in acute lung injury. A high-fat diet increases the severity of hyperoxia-induced acute lung injury in mice in part by altering FASN levels in the lung of high-fat diet-fed rodents. This is the first study to our knowledge to demonstrate that a high-fat diet leads to altered FASN expression in the lung and that both a high-fat diet and reduced FASN in alveolar epithelial cells lead to increased lung injury. A complex interaction exists among diet, weight, and lung function in the setting of hyperoxia that is unlikely to be explained by a single mechanism; FASN downregulation is likely not the only process promoting modified responses with a high-fat diet. Delineating individual pathways contributing to the final injury, however, may help identify obese patients who are at higher risk for lung complications in the setting of critical illness and aid in the implementation of preventive and therapeutic approaches.

\section{Methods}

Further information can be found in Supplemental Methods, available online with this article.

Experimental animals, obesity model, and exposure to hyperoxia. Four-week-old male C57BL/6J mice and 13-week-old male mice homozygous for the obese spontaneous mutation Lep ${ }^{o b}$ (B6.Cg-Lep $\left.{ }^{o b} / \mathrm{J}, o b / o b\right)$ and heterozygous controls $(o b /+)$ were purchased from the Jackson Laboratory. FASN $N^{\text {toxp } / \text { oxp }}$ mice were provided by Clay F. Semenkovich (Washington University School of Medicine in St. Louis) (36) and Sftp ${ }^{\text {CreERT2+/+ }}$ mice by Brigid Hogan (Duke University, Durham, North Carolina, USA) (35). To generate male mice with tamoxifen-inducible FASN deletion specifically in alveolar epithelial type II cells, FASN ${ }^{\text {toxp/loxp }}$ mice were crossed to Sftpc CreERT2+/+ mice. Sftpc $c^{\text {reERT2+/- }}$ mice were used as controls for experiments. At the age of 6 weeks, mice were divided into 2 groups. One group (obese group) received a diet in which $60 \%$ of the calories were derived from fat in the form of lard (D12492, OpenSource Diets) and the other group (control group) an ingredient-matched diet in which $10 \%$ of the calories was derived from fat (D12450B) for 12-14 weeks. Mice were exposed to hyperoxia in an airtight, custom-built plexiglass exposure chamber maintained with $18 \mathrm{~L} /$ min oxygen $\left(\mathrm{O}_{2}\right)\left(>95 \% \mathrm{O}_{2}\right)$.

Sample collection. BALF was collected by slowly washing the lungs with PBS via the endotracheal tube. BALF supernatant was used for measurement of total protein (Pierce BCA Protein Assay Kit, Thermo Fisher Scientific), IL-6 (IL-6 mouse uncoated ELISA kit, Thermo Fisher Scientific), TNFA (TNFA mouse uncoated ELISA kit, Thermo Fisher Scientific), and IgM (IgM mouse uncoated ELISA kit, Thermo Fisher Scientific) concentration, and free fatty acid quantification (Free Fatty Acid Quantification Colorimetric/ Fluorometric Kit, BioVision). The pellet was resuspended, total concentration of cells determined, and differential counts of inflammatory cells performed. The lungs were resected for immunoblotting. For histology, in a different set of animals, to maintain architecture the lung was distended through the trachea with $0.5 \%$ low-melting agarose and placed into cold $4 \%$ paraformaldehyde (PFA) (57). For RNA sequencing, the lungs were resected and placed in RNAlater (Sigma-Aldrich).

Serum cholesterol and fasting glucose measurements. Concentration of total serum cholesterol was determined spectrophotometrically on a Beckman Coulter AU analyzer (OSR6116, Beckman Coulter). Blood glucose levels were measured by placing a drop of blood onto a glucose strip (Accu-Chek, Roche) into a glucometer (Accu-Chek Aviva, Roche).

Cell death assays. Cell death was assessed by measuring LDH in BALF supernatant using a commercially available assay (LDH-Cytotoxicity Colorimetric Assay Kit II, BioVision) and by TUNEL staining on histology slides (58). 
Measurement of lung tissue free fatty acids. Lipids were extracted from lung tissue and dissolved in $3 \%$ Triton-X (59). Concentrations of free fatty acids were measured enzymatically in total lung lipid extracts (Wako Diagnostics).

RNA isolation and RNA-Seq analysis. RNA samples were obtained from lung tissue of C57BL/6J mice fed high-fat and control diets exposed to room air and hyperoxia. RNA was extracted using the RNeasy Mini Kit (QIAGEN) and submitted to the Genomic Resource Core Facility of Weill Cornell Medical College. Only high-quality RNA samples with a 260:280 ratio >1.6 and a RNA integrity number RIN $>7$ were used for the library construction using the TruSeq Stranded mRNA Library Preparation kit (Illumina). The sequencing was performed on the HiSeq4000 sequencer (Illumina). The abundance of transcripts was measured as fragments per kilobase of transcript per million fragments sequenced $(\mathrm{FPKM})(60,61)$. We compared the genes that were significantly different between high-fat and control diet mice with mouse MitoCarta2.0, an inventory of mouse genes encoding proteins with strong support of mitochondrial localization based on integrated proteomics, computation, and microscopy (https://www.broadinstitute.org/scientific-community/science/programs/metabolic-disease-program/publications/mitocarta/mitocarta-in-0) (62). RNA-Seq data are available under the accession GSE123938 in the NCBI's Gene Expression Omnibus database.

Isolation of AEC2 cells from mouse lungs through MACS separation. The isolation of AEC2 cells from mouse lungs was performed as previously described (63). Briefly, mouse lungs were perfused through the right ventricle, inflated by $1.5 \mathrm{~mL}$ BD Dispase (Biosciences) and $0.5 \mathrm{~mL} \mathrm{1 \%} \mathrm{low-melting} \mathrm{point} \mathrm{agarose} \mathrm{(Invitrogen),}$ and then transferred to dispase. After digestion, the lungs were homogenized manually with DMEM containing $200 \mathrm{U} / \mathrm{mL}$ DNase (Sigma-Aldrich). After filtration and centrifugation, the whole lung cell suspension was obtained and was negatively selected by CD45 microbeads (Miltenyi Biotec). This was followed by positive selection by streptavidin microbeads (Miltenyi Biotec) and biotin-conjugated anti-EpCAM antibody (eBioscience), through MACS separation columns.

To quantify the AEC2 cell purity in MACS-isolated CD45-EpCAM ${ }^{+}$populations, we performed immunofluorescence staining of surfactant protein C (SP-C). CD45-EpCAM ${ }^{+}$cells were fixed by $4 \%$ PFA and were transferred to slides by Cytospin centrifugation. The $\mathrm{CD}^{-} 5^{-} \mathrm{EPCAM}^{-}$population was used to prepare Cytospin slides for negative control. The cells were incubated overnight with primary antibody against SP-C (1: 1000 in blocking buffer, EMD Millipore ABC99). Sixteen to 24 hours later, the cells were incubated with the Alexa Fluor 488-conjugated secondary antibody (Thermo Fisher Scientific), and Hoechst 33342 (1:1000 dilution in TBS) was used to stain the nucleus. Images of the slides were obtained by confocal microscopy (Zeiss LSM 880 laser scanning microscope). The CD45-EpCAM ${ }^{+}$population was mainly composed of AEC2 cells (purity $94 \%$ ).

Western immunoblot analysis. Proteins were isolated from lung tissue samples and analyzed by Western blot using rabbit anti-mouse FASN (1:1000, Cell Signaling Technology), rabbit anti-claudin 18 (1:1000, Thermo Fisher Scientific), and mouse anti- $\beta$-actin (1:5000, Sigma-Aldrich). Immunoblotting was also performed with lysates of MACS-isolated AEC2 cells using Total OXPHOS Rodent WB Antibody Cocktail (1:1000, Abcam), mitochondrial membrane integrity WB antibody cocktail (1:1000, Abcam), and TOM20 (1:2000, Santa Cruz Biotechnology Inc.). The densitometry of the bands was measured using Fiji running ImageJ software (version 1.52b) (https://fiji.sc/) and was normalized to $\beta$-actin as a loading control.

Histology and IHC. For histological examination, murine lungs were submitted to the core laboratory at Weill Cornell Pathology and Laboratory Medicine for tissue processing, paraffin embedding, hematoxylin and eosin, and IHC staining. The primary antibodies against FASN (1:500, Cell Signaling Technology) and PCNA (1:2500, Abcam) were used for IHC

Seahorse analysis. Extracellular acidification rates (ECAR) and oxygen consumption rates (OCR) were determined by the Seahorse XF 96 flux analyzer (Seahorse Bioscience, Agilent Technologies). AEC2 cells were isolated, as described above, and they were plated overnight onto cell culture microplates (Seahorse Bioscience, Agilent Technologies) coated with $50 \mathrm{ng} / \mu \mathrm{L}$ Laminin 1 (Trevigen). Cells were incubated in XF assay medium (Seahorse Bioscience, Agilent Technologies), supplemented with $5 \mathrm{mM}$ glucose, $4 \mathrm{mM}$ glutamine and $1 \mathrm{mM}$ pyruvate for one hour prior to the measurement. After the recording of the basal rates of ECAR and OCR, final concentrations of $1 \mu \mathrm{M}$ oligomycin, $2 \mu \mathrm{M}$ carbonyl cyanide-4-(trifluoromethoxy) phenylhydrazone (FCCP), and $0.5-0.5 \mu \mathrm{M}$ rotenone and antimycin A were added (Sigma-Aldrich) through the instrument injection ports in order to obtain proton leak, maximal respiratory capacity, and nonmitochondrial respiration, respectively.

Statistics. Survival analysis between groups was calculated using the log-rank test. Continuous variables are presented as mean \pm SEM and compared with the nonparametric Mann Whitney $U$ test (for comparison of 2 groups) or ANOVA with Tukey's post hoc correction (for comparisons of more than 2 groups). 
A 2-tailed $P$ value of less than 0.05 was considered to denote statistical significance. All analyses were performed using GraphPad Prism 5.01.

Study approval. All experiments were approved by the Weill Cornell Medicine Institutional Animal Care and Use Committee.

\section{Author contributions}

MP designed the study, conducted experiments, acquired and analyzed data, and drafted the manuscript. LCF, ZH, LKT, MI, DEC, and ES performed experiments. YZ performed data analysis. SMC and AMKC designed experiments and participated in data analysis and manuscript preparation.

\section{Acknowledgments}

This work was supported by NIH grants R01 HL055330 (to AMKC); R00 HL125899 (to SMC); DK048873, DK056625, and DK103046 (to DEC); the Weill Cornell DOM Pre-Career Award (to MP); and China Scholarship Council no. 201706260177 (to ZH). The authors would like to thank Kristen T. Schiffer for technical support, Brigid L.M. Hogan at Duke University for sharing the $S f p c^{\text {CreERT2+/+ }}$ mice, and Clay F. Semenkovich at Washington University in St. Louis for sharing the Fasn ${ }^{\text {loxp/loxp }}$ mice. The authors also thank the Weill Cornell Medical Genomic Resources Core Facility and the Translational Research Program at Pathology and Laboratory Medicine.

Address correspondence to: Augustine M.K. Choi, Weill Cornell Medical College, 1300 York Avenue, Suite F-113 Box 83, New York, NY 10065, USA. Phone: 212.746.6005; Email: amc2056@med.cornell.edu.

LCF's present address is: Department of Respiratory and Critical Care Medicine, Shanghai Pulmonary Hospital, Tongji University School of Medicine, Shanghai, China.

MI's present address is: Department of Internal Medicine, St. Marianna University School of Medicine, Kawasaki, Japan.

YZ's present address is: Peking University Health Science Center, Beijing, China.

1. GBD 2015 Obesity Collaborators, et al. Health effects of overweight and obesity in 195 countries over 25 years. $N$ Engl J Med. 2017;377(1):13-27.

2. Hales CM, Carroll MD, Fryar CD, Ogden CL. Prevalence of obesity among adults and youth: United States, 2015-2016. NCHS data brief. 2017;(288):1-8.

3. Peters U, Suratt BT, Bates JHT, Dixon AE. Beyond BMI: obesity and lung disease. Chest. 2018;153(3):702-709.

4. Pépin JL, Timsit JF, Tamisier R, Borel JC, Lévy P, Jaber S. Prevention and care of respiratory failure in obese patients. Lancet Respir Med. 2016;4(5):407-418.

5. Mahul M, et al. Spontaneous breathing trial and post-extubation work of breathing in morbidly obese critically ill patients. Crit Care. 2016;20(1):346.

6. ARDS Definition Task Force, et al. Acute respiratory distress syndrome: the Berlin Definition. JAMA. 2012;307(23):2526-2533.

7. Bellani G, et al. Epidemiology, patterns of care, and mortality for patients with acute respiratory distress syndrome in intensive care units in 50 countries. JAMA. 2016;315(8):788-800.

8. Sakr Y, et al. Obesity is associated with increased morbidity but not mortality in critically ill patients. Intensive Care Med. 2008;34(11):1999-2009.

9. Ray DE, Matchett SC, Baker K, Wasser T, Young MJ. The effect of body mass index on patient outcomes in a medical ICU. Chest. 2005;127(6):2125-2131.

10. Lewandowski K, Lewandowski M. Intensive care in the obese. Best Pract Res Clin Anaesthesiol. 2011;25(1):95-108.

11. Gong MN, Bajwa EK, Thompson BT, Christiani DC. Body mass index is associated with the development of acute respiratory distress syndrome. Thorax. 2010;65(1):44-50.

12. Anzueto A, et al. Influence of body mass index on outcome of the mechanically ventilated patients. Thorax. 2011;66(1):66-73

13. De Jong A, et al. Impact of the driving pressure on mortality in obese and non-obese ARDS patients: a retrospective study of 362 cases. Intensive Care Med. 2018;44(7):1106-1114.

14. De Jong A, et al. Feasibility and effectiveness of prone position in morbidly obese patients with ARDS: a case-control clinical study. Chest. 2013;143(6):1554-1561.

15. Stapleton RD, Suratt BT. Obesity and nutrition in acute respiratory distress syndrome. Clin Chest Med. 2014;35(4):655-671.

16. Ball L, Serpa Neto A, Pelosi P. Obesity and survival in critically ill patients with acute respiratory distress syndrome: a paradox within the paradox. Crit Care. 2017;21(1):114.

17. Ware LB, Matthay MA. The acute respiratory distress syndrome. N Engl J Med. 2000;342(18):1334-1349.

18. Bachofen M, Weibel ER. Structural alterations of lung parenchyma in the adult respiratory distress syndrome. Clin Chest Med. 1982;3(1):35-56. 
19. Crapo JD. Morphologic changes in pulmonary oxygen toxicity. Annu Rev Physiol. 1986;48:721-731.

20. Kallet RH, Matthay MA. Hyperoxic acute lung injury. Respir Care. 2013;58(1):123-141.

21. Branson RD, Robinson BR. Oxygen: when is more the enemy of good? Intensive Care Med. 2011;37(1):1-3.

22. Eastwood G, et al. Arterial oxygen tension and mortality in mechanically ventilated patients. Intensive Care Med. 2012;38(1):91-98.

23. LaFemina MJ, et al. Claudin-18 deficiency results in alveolar barrier dysfunction and impaired alveologenesis in mice. Am J Respir Cell Mol Biol. 2014;51(4):550-558.

24. LaFemina MJ, et al. Keratinocyte growth factor enhances barrier function without altering claudin expression in primary alveolar epithelial cells. Am J Physiol Lung Cell Mol Physiol. 2010;299(6):L724-L734.

25. Niimi T, et al. Claudin-18, a novel downstream target gene for the T/EBP/NKX2.1 homeodomain transcription factor, encodes lung- and stomach-specific isoforms through alternative splicing. Mol Cell Biol. 2001;21(21):7380-7390.

26. Ahmad R, Rah B, Bastola D, Dhawan P, Singh AB. Obesity-induces organ and tissue specific tight junction restructuring and barrier deregulation by Claudin switching. Sci Rep. 2017;7(1):5125.

27. Mantell LL, Horowitz S, Davis JM, Kazzaz JA. Hyperoxia-induced cell death in the lung — the correlation of apoptosis, necrosis, and inflammation. Ann N Y Acad Sci. 1999;887:171-180.

28. Suratt BT. Mouse modeling of obese lung disease. Insights and caveats. Am J Respir Cell Mol Biol. 2016;55(2):153-158.

29. Lutz TA, Woods SC. Overview of animal models of obesity. Curr Protoc Pharmacol. 2012; Chapter 5:Unit 5.61.

30. Tzameli I. The evolving role of mitochondria in metabolism. Trends Endocrinol Metab. 2012;23(9):417-419.

31. Islam MN, et al. Mitochondrial transfer from bone-marrow-derived stromal cells to pulmonary alveoli protects against acute lung injury. Nat Med. 2012;18(5):759-765.

32. Budinger GR, et al. Epithelial cell death is an important contributor to oxidant-mediated acute lung injury. Am J Respir Crit Care Med. 2011;183(8):1043-1054.

33. Menendez JA, Vazquez-Martin A, Ortega FJ, Fernandez-Real JM. Fatty acid synthase: association with insulin resistance, type 2 diabetes, and cancer. Clin Chem. 2009;55(3):425-438.

34. Chirala SS, et al. Fatty acid synthesis is essential in embryonic development: fatty acid synthase null mutants and most of the heterozygotes die in utero. Proc Natl Acad Sci U S A. 2003;100(11):6358-6363.

35. Rock JR, et al. Multiple stromal populations contribute to pulmonary fibrosis without evidence for epithelial to mesenchymal transition. Proc Natl Acad Sci U S A. 2011;108(52):E1475-E1483.

36. Lodhi IJ, et al. Peroxisomal lipid synthesis regulates inflammation by sustaining neutrophil membrane phospholipid composition and viability. Cell Metab. 2015;21(1):51-64.

37. Afshin A, Reitsma MB, Murray CJL. Health effects of overweight and obesity in 195 countries. N Engl J Med. 2017;377(15):1496-1497.

38. Martino JL, et al. Extreme obesity and outcomes in critically ill patients. Chest. 2011;140(5):1198-1206

39. Morris AE, Stapleton RD, Rubenfeld GD, Hudson LD, Caldwell E, Steinberg KP. The association between body mass index and clinical outcomes in acute lung injury. Chest. 2007;131(2):342-348.

40. Flegal KM, Ioannidis JPA. The obesity paradox: a misleading term that should be abandoned. Obesity (Silver Spring). 2018;26(4):629-630

41. Wilson MR, et al. High-fat feeding protects mice from ventilator-induced lung injury, via neutrophil-independent mechanisms Crit Care Med. 2017;45(8):e831-e839.

42. Guivarch E, et al. Pulmonary effects of adjusting tidal volume to actual or ideal body weight in ventilated obese mice. Sci Rep. 2018;8(1):6439

43. Kordonowy LL, et al. Obesity is associated with neutrophil dysfunction and attenuation of murine acute lung injury. $A m J$ Respir Cell Mol Biol. 2012;47(1):120-127.

44. Shah D, et al. Obesity-induced adipokine imbalance impairs mouse pulmonary vascular endothelial function and primes the lung for injury. Sci Rep. 2015;5:11362.

45. Bellmeyer A, Martino JM, Chandel NS, Scott Budinger GR, Dean DA, Mutlu GM. Leptin resistance protects mice from hyper oxia-induced acute lung injury. Am J Respir Crit Care Med. 2007;175(6):587-594.

46. Soares NP, et al. The effects of the combination of a refined carbohydrate diet and exposure to hyperoxia in mice. Oxid Med Cell Longev. 2016;2016:1014928.

47. Siempos II, et al. RIPK3 mediates pathogenesis of experimental ventilator-induced lung injury. JCI Insight. 2018;3(9):97102.

48. Syed MA, Shah D, Das P, Andersson S, Pryhuber G, Bhandari V. TREM-1 attenuates RIPK3-mediated necroptosis in hyperoxia-induced lung injury in neonatal mice. Am J Respir Cell Mol Biol. 2019;60(3):308-322.

49. Han $\mathrm{CH}$, et al. Oxidative stress induced necroptosis activation is involved in the pathogenesis of hyperoxic acute lung injury. Biochem Biophys Res Commun. 2018;495(3):2178-2183.

50. Jensen-Urstad AP, Semenkovich CF. Fatty acid synthase and liver triglyceride metabolism: housekeeper or messenger? Biochim Biophys Acta. 2012;1821(5):747-753.

51. Stoops JK, Ross P, Arslanian MJ, Aune KC, Wakil SJ, Oliver RM. Physicochemical studies of the rat liver and adipose fatty acid synthetases. J Biol Chem. 1979;254(15):7418-7426.

52. Semenkovich CF, Coleman T, Fiedorek FT. Human fatty acid synthase mRNA: tissue distribution, genetic mapping, and kinetics of decay after glucose deprivation. J Lipid Res. 1995;36(7):1507-1521.

53. Treutlein B, et al. Reconstructing lineage hierarchies of the distal lung epithelium using single-cell RNA-seq. Nature. 2014;509(7500):371-375.

54. Moon YS, Latasa MJ, Griffin MJ, Sul HS. Suppression of fatty acid synthase promoter by polyunsaturated fatty acids. J Lipid Res. 2002;43(5):691-698.

55. Wei X, et al. De novo lipogenesis maintains vascular homeostasis through endothelial nitric-oxide synthase (eNOS) palmitoylation. J Biol Chem. 2011;286(4):2933-2945.

56. Wei X, et al. Fatty acid synthase modulates intestinal barrier function through palmitoylation of mucin 2. Cell Host Microbe. 2012;11(2):140-152.

57. Halbower AC, Mason RJ, Abman SH, Tuder RM. Agarose infiltration improves morphology of cryostat sections of lung. Lab 
Invest. 1994;71(1):149-153.

58. Gavrieli Y, Sherman Y, Ben-Sasson SA. Identification of programmed cell death in situ via specific labeling of nuclear DNA fragmentation. J Cell Biol. 1992;119(3):493-501.

59. Tillander V, Miniami A, Alves-Bezerra M, Coleman RA, Cohen DE. Thioesterase superfamily member 2 promotes hepatic insulin resistance in the setting of glycerol-3-phosphate acyltransferase 1-induced steatosis. J Biol Chem. 2019;294(6):2009-2020.

60. Trapnell C, Hendrickson DG, Sauvageau M, Goff L, Rinn JL, Pachter L. Differential analysis of gene regulation at transcript resolution with RNA-seq. Nat Biotechnol. 2013;31(1):46-53.

61. Trapnell C, et al. Transcript assembly and quantification by RNA-Seq reveals unannotated transcripts and isoform switching during cell differentiation. Nat Biotechnol. 2010;28(5):511-515.

62. Calvo SE, Clauser KR, Mootha VK. MitoCarta2.0: an updated inventory of mammalian mitochondrial proteins. Nucleic Acids Res. 2016;44(D1):D1251-D1257.

63. Messier EM, Mason RJ, Kosmider B. Efficient and rapid isolation and purification of mouse alveolar type II epithelial cells. Exp Lung Res. 2012;38(7):363-373. 